\title{
Decreased expression of TERT correlated with postnatal cochlear development and proliferation reduction of cochlear progenitor cells
}

\author{
YONG-LI SONG ${ }^{*}$, KE-YONG TIAN* , WEN-JUAN MI, ZHONG-JIA DING, \\ YANG QIU, FU-QUAN CHEN, DING-JUN ZHA and JIAN-HUA QIU \\ Department of Otolaryngology-Head and Neck Surgery, Xijing Hospital, \\ Air Force Military Medical University, Xi'an, Shaanxi 710032, P.R. China
}

Received September 24, 2017; Accepted January 17, 2018

DOI: $10.3892 / \mathrm{mmr} .2018 .8565$

\begin{abstract}
Cochlear progenitor cells are considered as one of the best candidates for hair cell regeneration, thus, the regulation of cochlear progenitor cell proliferation has become a focus in this field. Several genes expressed in the inner ear during postnatal development have been demonstrated to be involved in maintaining the proliferative potential of progenitor cells, but the mechanism for regulating the proliferation and differentiation of cochlear progenitor cells remains poorly understood. Telomerase reverse transcriptase (TERT) has rate limiting telomerase activity and the overexpression of TERT has been shown to promote cell proliferation in series of cell lines. The aim of the present study was to evaluate the expression of TERT in the postnatal development of the cochlea and progenitor cells. The results demonstrated that TERT was expressed in the basilar membranes during the first postnatal week. In vitro, TERT expression in progenitor cells reached a maximum at day 4 after culture and decreased as the culture time prolonged or the cell passage number increased. These results led us to hypothesize that TERT may be involved in the development of the cochlea and in maintaining the proliferation ability of progenitor cells.
\end{abstract}

\section{Introduction}

The lack of adult mammalian hair cell regeneration after damage leads to permanent hearing loss, which for years has

Correspondence to: Professor Ding-Jun Zha or Jian-Hua Qiu, Department of Otolaryngology-Head and Neck Surgery, Xijing Hospital, Air Force Military Medical University, 127 Changle Western Road, Xi'an, Shaanxi 710032, P.R. China

E-mail: qiujh@fmmu.edu.cn; zhadjun@fmmu.edu.cn

\section{${ }^{*}$ Contributed equally}

Key words: cochlear progenitor cells, proliferation, TERT, hair cells, cell culture attracted attention to manipulating stem/progenitor cells to participate in hair cell regeneration. To date, cells dissociated from the neonatal mammalian basilar membrane have been proved to re-enter the cell cycle and have shown a limited ability to proliferate and differentiate into hair cells in vitro (1).

Typically, only a small fraction of the dissociated cells is able to grow into colonies and ultimately give rise to hair cells. Sinkkonen et al purified four different non-sensory cell populations from neonatal mouse cochleae by fluorescence-activated cell sorting (FACS). They found that cochlear-supporting cells and cells of the lesser epithelial ridge showed robust potential to proliferate and differentiate into hair cells (2). Shi et al found that Lgr5-expressing supporting cells, as sorted by flow cytometry, displayed enhanced capacity for self-renewing neurosphere formation in response to Wnt and were converted into hair cells at a higher ( $>10$-fold) rate than unsorted cells were. Lgr5-positive cells had the capacity to act as cochlear progenitor cells, and lineage tracing confirmed that Lgr5-expressing cells accounted for the cells that formed neurospheres and differentiated into hair cells (3). Furthermore, the proliferative ability of dissociated cochlear cells is transient, and it ceased after the second postnatal week in mice $(4,5)$. Several genes expressed in the inner ear during postnatal development were proved to be involved in maintaining the proliferative potential of progenitor cells, but the mechanism for regulating the proliferation and differentiation of cochlear progenitor cells remains relatively poorly understood.

Telomerase, a reverse transcriptase in eukaryocytes, is active in most stem cells, tumor cells and embryonic tissues. The main function of telomerase is to maintain the integrity of chromosomes and the cell cycle. The telomerase complex is composed of two main subunits that provide the enzymatic core function: Telomerase reverse transcriptase (TERT) and an RNA component (TERC). TERC is ubiquitously expressed in normal somatic cells, which serve as a template for telomeric DNA synthesis. TERT expression is limited to highly proliferative tissues; thus, the presence of TERT is rate limiting for telomerase activity (6). The overexpression of TERT could promote proliferation and immortalization in several series of cells $(7,8)$, but the role of TERT in the inner ear remains unclear. 
In this study, we evaluated the expression of TERT in the cochlear basilar membrane during postnatal development (P0, P3, P7, P14, P28). Further, the differential TERT levels in cochlear progenitor cells of different culture times, in different otospheres with distinct morphologies and in different generations were evaluated by RT-qPCR. This study allowed us to evaluate for the first time the role of TERT in the development of the cochlea and the proliferation process of cochlear progenitors, thereby providing evidence for the application of TERT in regulating cochlear progenitor cell proliferation and hair cell regeneration.

\section{Materials and methods}

Animals and cochlear dissection. Sprague-Dawley (SD) rats were provided by the Laboratory Animal Center of the Air Force Military Medical University. Experiments were conducted under protocols approved by the Animal Care and Use Committee of the Air Force Military Medical University.

$\mathrm{P} 0$ rats were anesthetized on ice, and the others were deeply anesthetized by intraperitoneally administration of an over-dose of choral hydrate $(45 \mathrm{mg} / \mathrm{kg})$. After anesthesia, rats at different ages (P0, P3, P7, P14, P28) were sacrificed by cervical dislocation, and the skulls were opened midsagitally. Using a dissecting microscope (SZX10; Olympus, Tokyo, Japan), the cochleae were dissected from the temporal bone followed by removal of the otic bulla to visualize the otic capsule. For ex vivo culture, the membranous labyrinth from P0-P3 rats was exposed, and the cochlear duct was retrieved after excision of the cartilage. The cochlear basilar membranes were micro-dissected from Reissner's membrane, the spiral ligament, and the stria vascularis and were then washed in ice-cold Hank's Balanced Salt Solution (HBSS; Invitrogen; Thermo Fisher Scientific, Inc., Waltham, MA, USA) and collected for further use.

For in vitro cell culturing, the harvested sheets were inspected, rinsed in sterile, chilled Hanks' balanced salt solution, and processed for cell dissociation.

Cell dissociation and sphere generation. The basilar membranes were incubated in D-Hanks' solution containing $0.5 \mathrm{mg} / \mathrm{ml}$ thermolysin (Sigma-Aldrich; Merck KGaA, Darmstadt, Germany) for $20 \mathrm{~min}$ at $37^{\circ} \mathrm{C}$, and the digestive enzymes were blocked by $10 \%$ fetal bovine serum in Dulbecco's modified Eagle's medium/F12 medium (Gibco; Thermo Fisher Scientific, Inc.). The tissue was triturated carefully 30-50 times with glass pipette tips. The suspension was passed through a $70 \mu \mathrm{m}$ cell trainer to remove clumps and cellar aggregates. The collected cells were cultured at a density of $1 \times 10^{4}$ cells $/ \mathrm{ml}$ in serum-free medium consisting of DMEM/F12 (Gibco; Thermo Fisher Scientific, Inc.), supplemented with $\mathrm{N} 2$ and B27, bFGF (10 ng/ml), and ampicillin (50 $\mu \mathrm{g} / \mathrm{ml}$ ) (all from Sigma-Aldrich; Merck KGaA) in 24-well suspension culture plates. The otospheres were collected at days 2, 4, and 7 for study. The morphological description of sphere formation was the same as previously reported (4). Briefly, the morphologies of the three types of otospheres were distinguished, the numbers were counted under a light microscope (5 wells) at days 2, 4, and 7, and the ratio of each type of otosphere was calculated. For propagation, otospheres were collected and dissociated from the cells mechanically after treatment with $0.125 \%$ trypsin at $37^{\circ} \mathrm{C}$ for $5 \mathrm{~min}$, and the cells were reflated in sphere culture medium for 3-5 days to obtain a second generation.

Cell proliferative capability evaluation. 3-(4,5-dimethylthiazol-2-yl)-2,5-diphenyltetrazolium bromide (MTT) solution (MTT assay kit; Sigma-Aldrich; Merck KGaA) was applied to evaluate the cell proliferation rates in vitro culture. Briefly, the cells dissociated from basilar epithelial sheets and were plated in 96-well dishes at 3,000 cells/well. The time-points for MTT assay were from day 1 to 7 after culture. After the predetermined time-points of incubation, $10 \mu \mathrm{l}$ of $10 \mathrm{mg} / \mathrm{ml}$ MTT solution was added and incubated for $4 \mathrm{~h}$. Then, the medium was removed, and $150 \mu \mathrm{l}$ dimethyl sulfoxide (DMSO) was added, followed by shaking for $10 \mathrm{~min}$. The optical density of the solutions in the wells was measured at $490 \mathrm{~nm}$ using a photometer (MK3 Multilabel Plate Reader; Thermo Fisher Scientific, Inc.).

Immunocytochemistry. The cochlear from P0, P7, P14, P28 were fixed with $4 \%$ paraformaldehyde in $0.1 \mathrm{M} \mathrm{PBS}$ for $6 \mathrm{~h}$ at room temperature and then dehydrated by $30 \%$ sucrose overnight, then the slices were prepared in $10 \mu \mathrm{m}$ of thickness. The cells were fixed with $4 \%$ paraformaldehyde in $0.1 \mathrm{M}$ PBS for $15 \mathrm{~min}$ at room temperature and were then washed in $0.01 \mathrm{M}$ PBS for $5 \mathrm{~min}$ three times. For immunofluorescent staining, the slices and fixed cells were treated with $1 \%$ Triton X-100 (Sigma-Aldrich; Merck KGaA) on ice for $5 \mathrm{~min}$. Non-specific binding sites were blocked for $1 \mathrm{~h}$ in PBS containing $0.2 \%$ Triton X-100 and 5\% bovine serum solution. Primary antibodies of mouse anti-nestin (1:100; Millipore, Billerica, MA, USA) and rabbit anti-TERT (1:50; Santa Cruz Biotechnology, Inc., Dallas, TX, USA) were incubated in PBS with 5\% BSA and $0.2 \%$ Triton $\mathrm{X}-100$ overnight at $4^{\circ} \mathrm{C}$. Then, the secondary antibodies conjugated with Alexa Flour 594 or 488 (1:500; Invitrogen; Thermo Fisher Scientific, Inc.) were added for $2 \mathrm{~h}$ at room temperature, and the nuclei were stained by DAPI (4',6-diamidino-2-phenylindole, 1:1,000; Sigma-Aldrich; Merck KGaA). Specimens were evaluated under a confocal microscope (Leica Microsystems GmbH, Wetzlar, Germany). Negative control experiments were performed as above by omitting the primary antibody.

$R T-q P C R$. Total RNA was isolated from cultured otospheres with RNeasy Micro kits, and $500 \mathrm{ng}$ of total RNA was used for reverse transcription (both from Qiagen, Valencia, CA, USA). Polymerase chain reaction (PCR) analysis was performed with an ABI PRISM 7500 fast real-time PCR system (Applied Biosystems, Foster City, CA, USA). The $20 \mu 1$ reaction mixture contained $10 \mu \mathrm{l}$ of $2 \mathrm{x}$ Green PCR Master Mix, $1 \mu \mathrm{l}$ of cDNA template, $1 \mu \mathrm{l}$ of each primer $(10 \mathrm{~nm})$, and $7 \mu \mathrm{l}$ of RNase-free water. The reaction was performed at $95^{\circ} \mathrm{C}$ for $5 \mathrm{~min}$, followed by 40 cycles of $95^{\circ} \mathrm{C}$ for $10 \mathrm{sec}$ and $60^{\circ} \mathrm{C}$ for $30 \mathrm{sec}$. A sample without cDNA template was processed in parallel and served as a negative control. All of the RT-qPCR reactions were performed in triplicate, with the resultant values combined into an average cycle threshold (cq). The $\Delta \mathrm{cq}$ method with GAPDH as the endogenous reference was used to determine the relative levels of gene expression. 


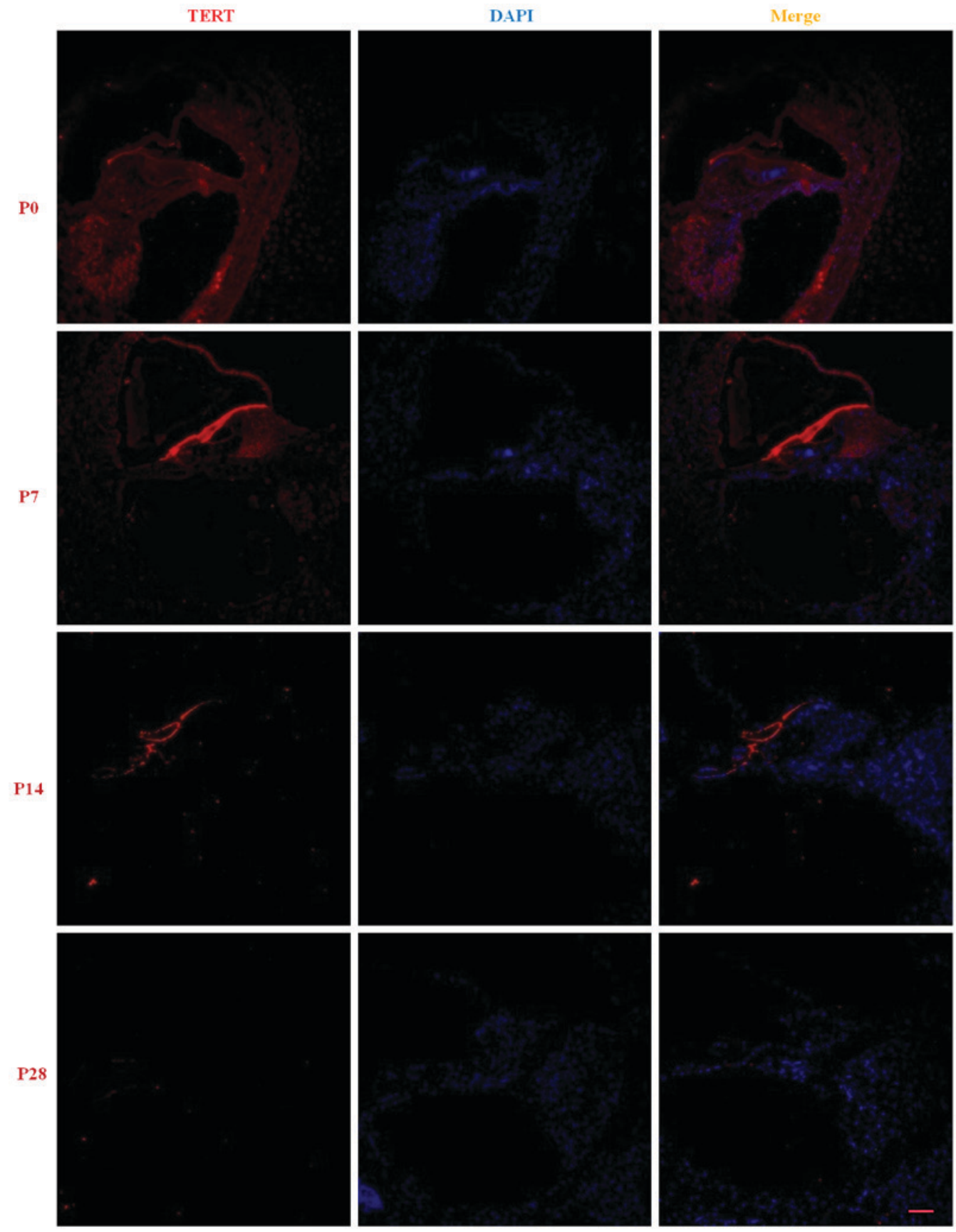

Figure 1. The expression of TERT in the cochlear during postnatal development (P0, P7, P14, P28) by immunofluorescence. There was low expression of TERT in basilar membrane, spiral ganglion and lateral wall of cochlea at P0 and the fluorescence gradually decreased as cochlear development. At P28, almost no gradually were observed (red; Scale bar, $50 \mu \mathrm{m}$ ). TERT, telomerase reverse transcriptase.

The primers were as follows: PCNA sense, 5'-GCAAC T TGGAATCCCAGAACAGG-3' and antisense, 5'-CGCAGA AAACTTCACCCCGTC-3'. Other primers were provided by GeneCopoeia, Rockville, MD, USA, including TERT (cat. no. RQP051634), nestin (cat. no. RPQ049303), and GAPDH (cat. no. RQP049537).

Western blot analysis. Isolated basilar membrane sheets were collected and homogenized in ice-cold protein lysis buffer (Sigma-Aldrich; Merck KGaA) containing $\mathrm{NaCl} 150 \mathrm{mM}$, Tris $50 \mathrm{mM}$, NP-40 1\%, DOC $0.5 \%$, SDS $0.1 \%$ and protein inhibitor cocktail $(1 \mathrm{mM})$. After incubation for $30 \mathrm{~min}$ on ice, the samples were centrifuged at $14,000 \mathrm{rpm}$ for $30 \mathrm{~min}$ at $4^{\circ} \mathrm{C}$.
The supernatant was transferred to a new tube. Protein was then collected for further use.

Protein samples were denatured in gel sample buffer [100 mM Tris-Cl (pH 6.8), 20\% glycerol, 4\% SDS, 200 mM DTT, and $0.2 \%$ bromophenol blue] by boiling for $5 \mathrm{~min}$ at $100^{\circ} \mathrm{C}$. An equivalent quantity of protein $(35 \mu \mathrm{g} /$ lane $)$ was separated on $10 \%$ SDS-polyacrylamide gels and was transferred to PVDF membranes (Roche Diagnostics, Basel, Switzerland). After blocking in 5\% bovine serum albumin, the membranes were incubated for $1 \mathrm{~h}$ at room temperature and then overnight at $4{ }^{\circ} \mathrm{C}$ with the following antibodies: Rabbit anti-TERT polyclonal antibody (1:200; Santa Cruz Biotechnology, Inc.) and rabbit anti-GAPDH polyclonal 

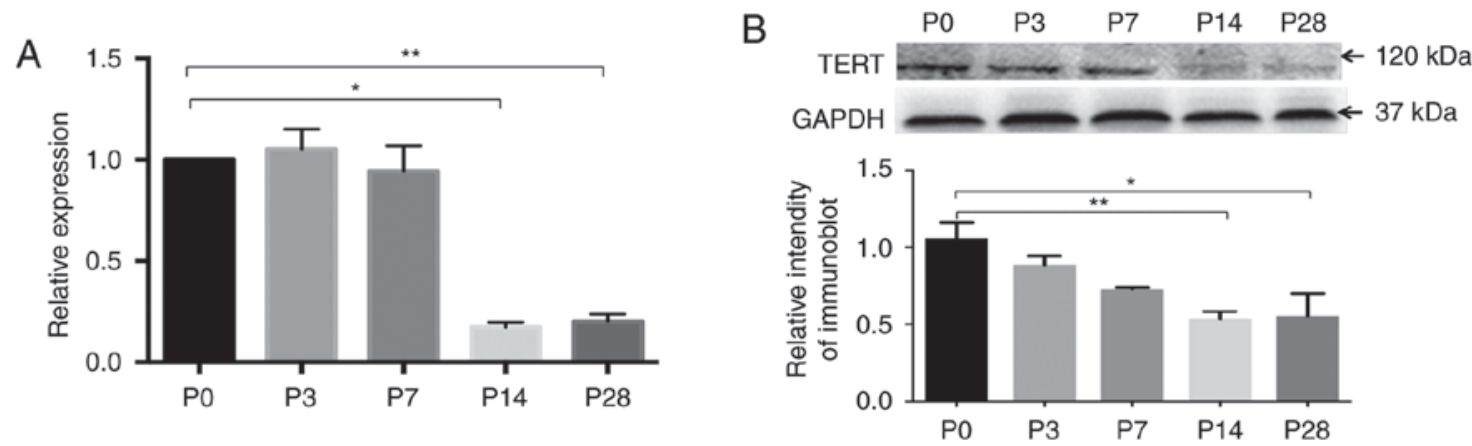

Figure 2. (A) RT-qPCR results of TERT mRNA expression in the basilar membrane during postnatal development. Relatively high levels of TERT mRNA were observed at $\mathrm{P} 0, \mathrm{P} 3$, and $\mathrm{P} 7$, which then significantly decreased to minimal levels at P14 and P28 ${ }^{*, * *} \mathrm{P}<0.05, \mathrm{n}=3$ ). (B) Western blot results of TERT protein expression in the basilar membrane during postnatal development. The principal immunoreactive band of $120 \mathrm{kDa}$ was detected with TERT antibody. The antibody GAPDH was used as a loading control. The TERT protein was expressed at the maximum level after birth (P0) but gradually declined during postnatal development and reached the lowest levels at P14 and P28 $\left({ }^{* * * *} \mathrm{P}<0.05, \mathrm{n}=5\right)$. TERT, telomerase reverse transcriptase.

antibody (1:00; Proteintech Group, Inc., Chicago, IL, USA). The membranes were then incubated with the peroxidase-labeled secondary antibodies (1:2,000; Santa Cruz Biotechnology, Inc.) at room temperature for $2 \mathrm{~h}$. The membranes were detected by enhanced chemiluminescence (ECL; Millipore). The band intensity was measured, and the value was normalized with regard to GAPDH. All experiments were repeated 5 times to ensure the reproducibility of the results.

Statistical analysis. Quantitative data are expressed as the means $\pm \mathrm{SD}$. The statistical process was examined by one-way analysis of variance (ANOVA), followed by the least significant difference (LSD) post hoc test. Analysis was performed using the Statistical Package for the Social Sciences (SPSS software, version 21.0; SPSS, Inc., Chicago, IL, USA). P<0.05 was considered to indicate a statistically significant difference.

\section{Results}

TERT expression in the cochlear basilar membrane during postnatal development. There was low expression of TERT in basilar membrane, Spiral ganglion and lateral wall of cochlea at $\mathrm{P} 0$ and the fluorescence gradually decreased as cochlear development (red). At P28, almost no gradually were observed (Fig. 1). The RT-qPCR results showed that relatively high levels of TERT mRNA in the basilar membrane were observed at P0, P3, and P7 and gradually decreased to a minimum level at P14 and P28 (Fig. 2A; P<0.05, n=3). The western blot results showed that the principal immunoreactive band of $120 \mathrm{kDa}$ was detected with TERT antibody. The antibody GAPDH was used as a loading control. The TERT protein was expressed at a maximum level after birth, but it gradually decreased during postnatal development, and the levels of TERT at P14 and P28 were significantly lower than that at $\mathrm{P} 0$ (Fig. $2 \mathrm{~B} ; \mathrm{P}<0.05, \mathrm{n}=3$ ).

\section{TERT expression in cochlear progenitor cells in vitro}

The proliferative rate of cochlear progenitor cells decreased as the culture time increased. We collected cultured cochlear otospheres at different times to evaluate the cell proliferation rate by MTT assay. We observed a significantly enhanced proliferation of cells from day 1 to 4 and found the maximum

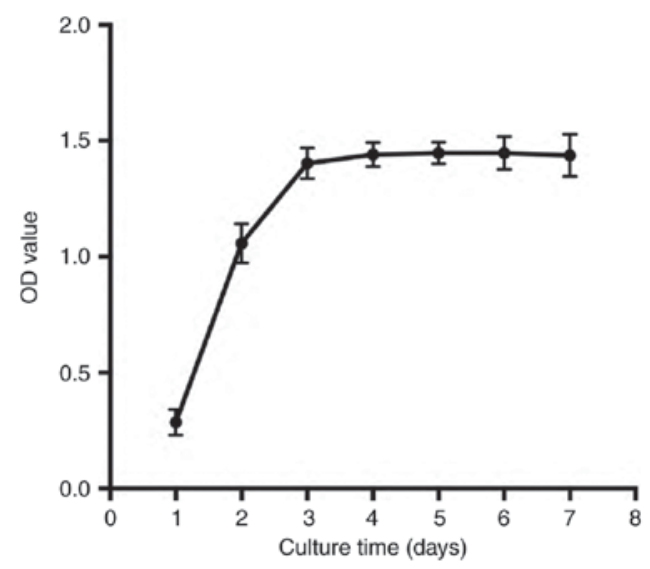

Figure 3. MTT assay results, which showed that significantly enhanced proliferation of cells was observed from day 1 to 4 and reached the maximum effect at day 4 . Then, the growth of cochlear progenitor cells remained stable from day 5 to $7(n=3)$.

effect at day 4 . Then, the growth of cochlear progenitor cells remained stable from day 5 to 7 (Fig. 3; n=3), which indicated that the proliferative rate of the cochlear progenitor cells was decreased.

The harvested cells could give rise to three different types of morphologically distinct spheres when cultured in vitro, and the ratio of each type of morphologically distinct sphere varied as the culture times prolonged. We compared the ratios of the three different otospheres at days 2,4 , and 7 . As shown in Fig. 4A, the volume of otospheres gradually increased as the culture time increased. The ratio of solid otospheres was maximal at day 2 and then decreased, whereas the ratio of hollow otospheres increased and reached the maximum ratio at day 7 ( Fig. 4B).

The expression of TERT in cochlear progenitor cells cultured in vitro. Immunocytochemical studies showed that cochlear progenitor cells were positive for TERT expression when cultured in vitro. The fluorescence intensity became stronger from day 2 to 4 , and the strongest fluorescence was observed at day 4, but almost no fluorescence was observed at day 7 (Fig. 5). RT-qPCR showed that the TERT mRNA expression was at the minimum level at day 2 , gradually increasing and 
A

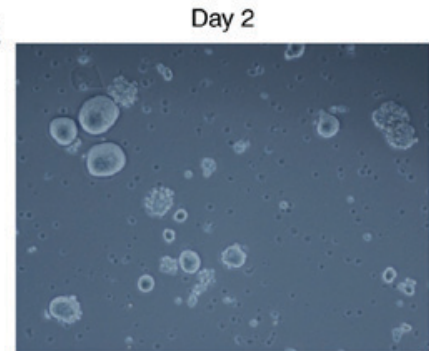

B

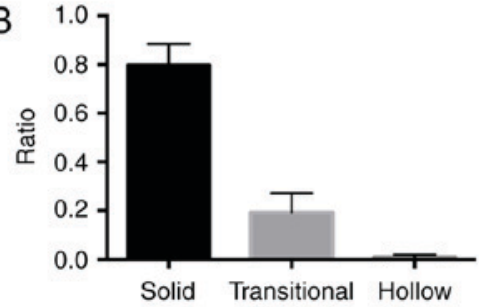

Day 4
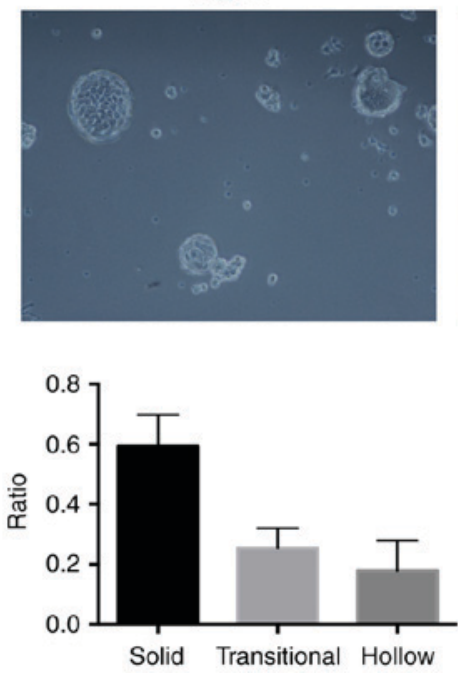

Day 7
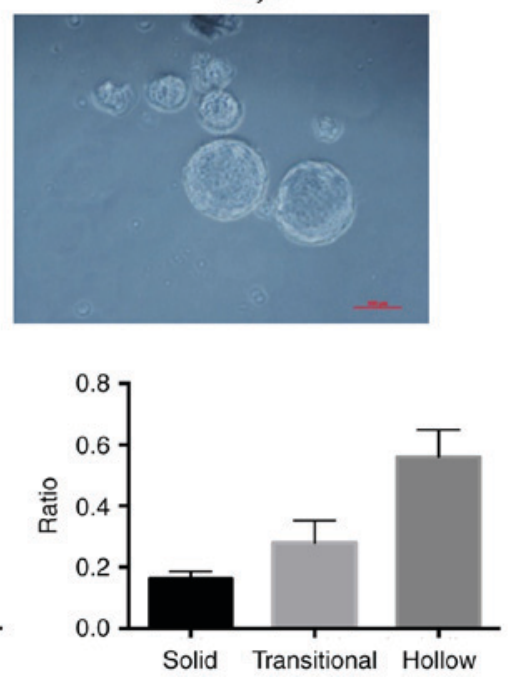

Figure 4. The features of morphology and the ratios of different otospheres of distinct morphology at different culture times. (A) Shows the morphology of otospheres formed on different days. At day 2, most of them were small, solid otospheres. At day 4, the otospheres became larger, and some hollow otospheres could be seen. At day 7, most of the otospheres were large, hollow otospheres (Scale bar, $100 \mu \mathrm{m}$ ). (B) Shows that the ratio of solid otospheres was maximal at day $2(79.6 \%)$ and then decreased, whereas the ratio of hollow otospheres increased and reached the maximum ratio at day 7 (17.6\%).
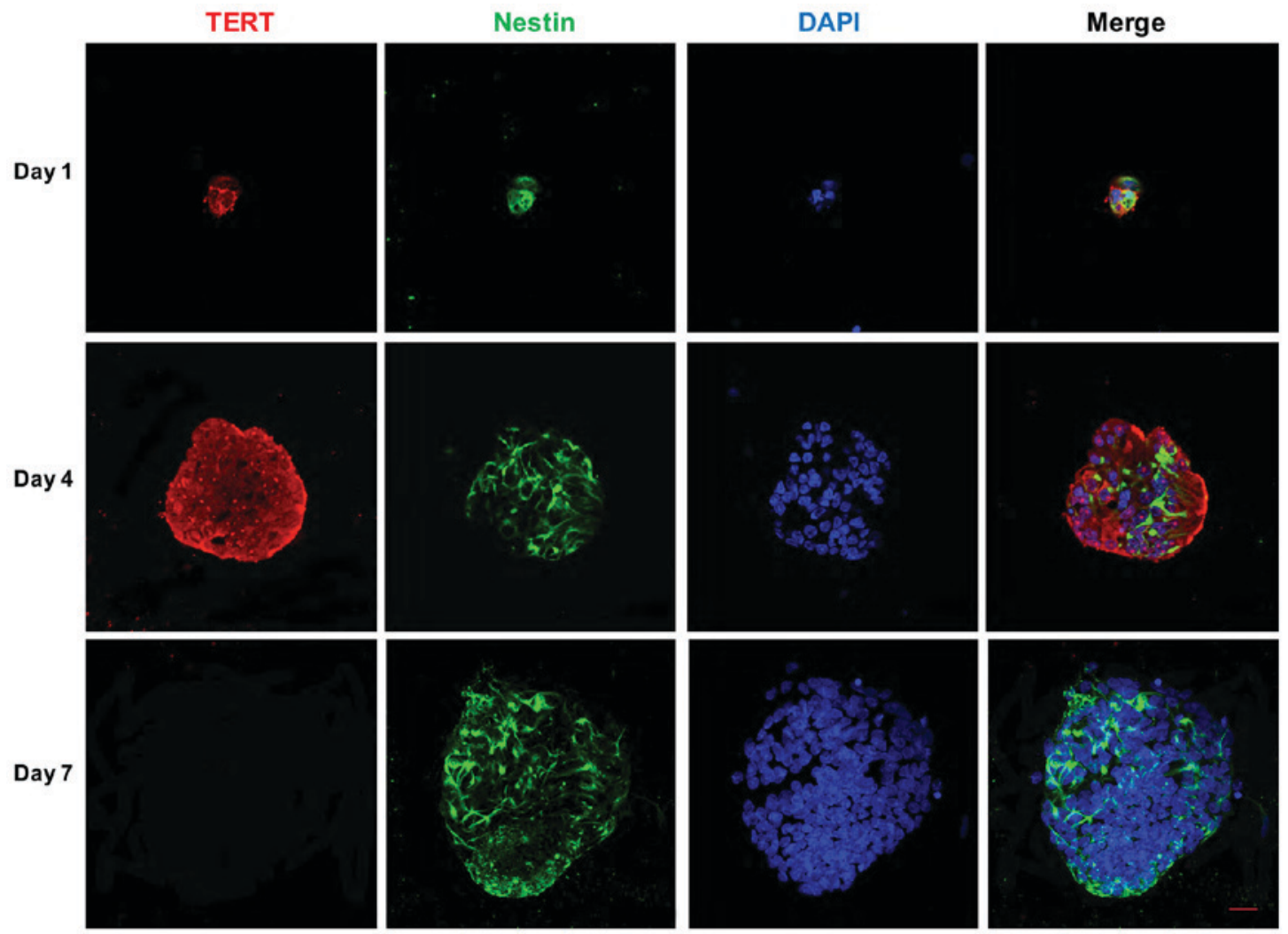

Figure 5. TERT expression in progenitor cells was observed both at nuclei and plasma (red). The fluorescence intensity of TERT became stronger from day 2 to 4 when cultured in vitro, and the strongest fluorescence was observed at day 4 , but almost no fluorescence was observed at day 7 . The expression of nestin did not show any significant difference (green; Scale bar, $20 \mu \mathrm{m}$ ). TERT, telomerase reverse transcriptase.

reaching at the maximum level at day 4 , coincident with the expression of nestin and PCNA ( $\mathrm{P}<0.05, \mathrm{n}=3)$ (Fig. 6A).

The otospheres with different morphologies were identified under a microscope on day 7 to evaluate the levels of nestin, PCNA and TERT mRNA among the three types of otospheres. The results showed that the level of TERT mRNA was significantly higher in solid otospheres than in the hollow otospheres $(\mathrm{P}<0.05, \mathrm{n}=3)$, whereas the expression of nestin and PCNA did not show any significant differences among the three types of otospheres ( $P>0.05, n=3$ ) (Fig. 6B).

As is known, the cochlear progenitor cells obtained limited proliferative capabilities when cultured in vitro, and the 
A

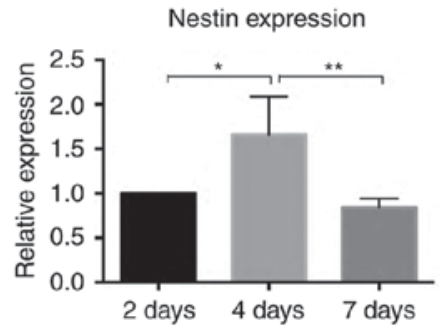

$\mathrm{B}$
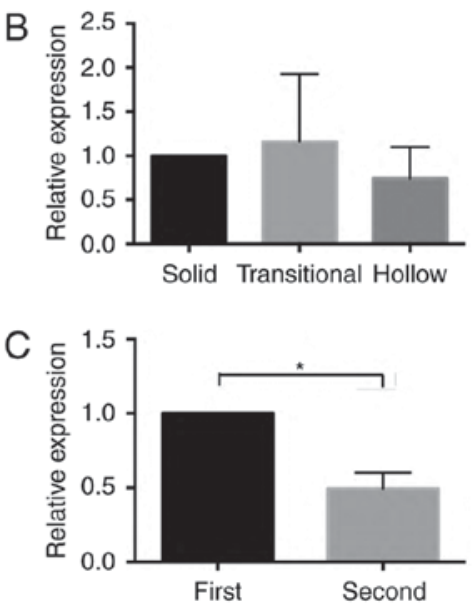
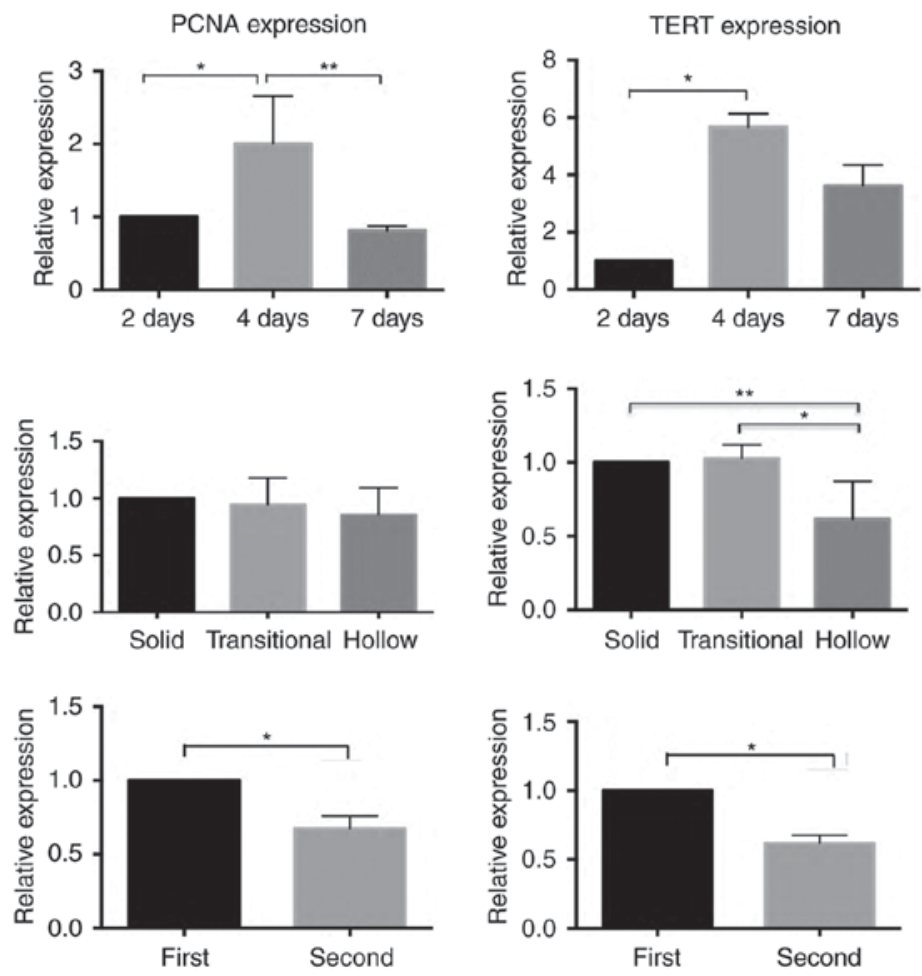

Figure 6. RT-qPCR results for nestin, PCNA, and TERT expression in progenitor cells at days 2, 4, and 7. The expression of nestin and PCNA attained the highest level at day $4\left({ }^{* * *} \mathrm{P}<0.05, \mathrm{n}=3\right)$; the expression of TERT was significantly higher at day 4 than at days 2 and 7 (A) $(* * * * 00.05$, $\mathrm{n}=3)$. RT-qPCR results of nestin, PCNA and TERT mRNA in three different types of morphological otospheres. The expression of nestin and PCNA did not show any significant differences among the three types of otospheres. The expression of TERT was significantly higher in solid and transitional otospheres than in hollow otospheres (B) $\left(* *{ }^{*} \mathrm{P}<0.05, \mathrm{n}=3\right)$. Differential expression of nestin, PCNA and TERT mRNA in the first and second generations of progenitor cells. The expressions of nestin, PCNA and TERT were significant higher in the first generation than in the second generation $(\mathrm{C})\left({ }^{*} \mathrm{P}<0.05, \mathrm{n}=3\right)$. TERT, telomerase reverse transcriptase.

proliferation capabilities decreased after cell passage. In our study, we collected the first and second generations of progenitor cells. The expression of TERT, nestin and PCNA was evaluated by RT-qPCR. The expression of TERT significantly decreased when the progenitor cells were passaged, coincident with the expression of nestin and PCNA ( $\mathrm{P}<0.05, \mathrm{n}=3$ ) (Fig. 6C).

\section{Discussion}

Cochlear progenitor cells are a subset of distinct cells between stem cells and terminally differentiated cells, and they obtain limited proliferative capability and exit the cell cycle after mitosis (9). Several genes involved in cochlear development have been proved to promote the proliferation of cochlear progenitor cells. Chai et al demonstrated that upregulating the level of $\beta$-catenin in progenitor cells could significantly promote cell proliferation (10), but that inhibiting the Notch pathway could promote progenitor cell differentiation into hair cells (11). However, the mechanism for the regulation of cochlear progenitor cell proliferation remains poorly understood.

In this study, we evaluated the expression of TERT in inner ear cells. First, we evaluated the differential expression levels of TERT in the basilar membrane during postnatal development by RT-qPCR and western blot analysis. Then, the TERT expression in the progenitor cells was evaluated by immunocytochemistry and RT-qPCR. The aim of this study was to evaluate the expression level and potential functions of TERT in postnatal cochlear development and in cochlear progenitor cells cultured in vitro. Collectively, our results could provide evidence for the presence of TERT in the postnatal cochlea in vivo and progenitor cells in vitro, suggesting a possible role of TERT in the regulation of postnatal cochlear development and progenitor cell proliferation.

TERT is the main component of telomerase complex, the presentation of which is critical to maintaining the activity of telomerase (12-17). Most eukaryocyte somatic cells have undetectable telomerase activity due to transcriptional repression of the catalytic subunit TERT during the early stage of embryo development, but its expression is limited to highly proliferative tissues, such as germ cells and stem cells after birth. Several studies have proved that TERT is critical for maintaining the capability of stem cells and promoting cell proliferation (18-24).

TERT was expressed in the basilar membrane during the first 7 days after birth, which indicated that TERT may be related to the postnatal development of the cochlea. As previously reported, the MTT assay results showed that progenitor cells proliferated significantly from day 1 to 4 and reached the maximum effect at day 4 . Then, the growth of cochlear progenitor cells remained stable from day 5 to 7 . The RT-qPCR results showed that nestin and PCNA were expressed at the highest levels at day 4. All of these results suggested that the proliferation capability of progenitor cells increased gradually and reached the maximum at day 4 . The TERT expression in the cochlear progenitor cells reached the maximum level at day 4 , coincident with the variety of proliferation abilities of progenitor cells, provided evidence 
that the expression of TERT might be involved in maintaining the proliferation capability of cochlear progenitor cells.

The progenitor cells cultured in vitro were observed to give rise to three different types of morphologies, that is, solid otospheres, transitional otospheres and hollow otospheres. Each type of otosphere obtained distinct features. The solid otospheres showed the smallest volume, and they were composed of small densely packed cells without any features of epithelial origin. In contrast, the hollow otospheres obtained the largest volume, and the cells were larger, with the classic characteristics of epithelial cells, coincident with previous reports. Whitlon reported their observation that the progenitor cells initially formed solid otospheres and that these otospheres became larger and transformed into hollow otospheres $48 \mathrm{~h}$ after culture, with the increasing volume of single cells. The cells in hollow otospheres formed a single layer organization, with increased expression of E-cadherin, which was localized to cellular junctions (25). All of these findings indicated a mature epithelial morphology. The cells from hollow otospheres could not form new spheres after they were digested into single cells, whereas cells from solid otospheres could give rise to new otospheres after passage, which indicated that the progenitor cells gradually lost the features and proliferative ability of stem cells as they transformed into hollow otospheres $(4,5,26)$. The RT-qPCR results showed that the nestin and PCNA mRNA levels were significantly higher in the solid otospheres and decreased after passage, indicating that the proliferative capacity of progenitor cells decreased as culture time and passage number increased, but the expression of TERT significantly decreased when the otospheres transformed from solid otospheres into hollow otospheres, and progenitor cells were passaged.

In conclusion, we demonstrated that TERT was expressed in the basilar membrane during the first 7 days after birth. The expression of TERT in progenitor cells reached the maximum at day 4 and decreased as both the culture time and number of passages increased, coincident with the proliferation variety of cochlear progenitor cells. All of these results led us to hypothesize that TERT might be involved in the development of the cochlea and in maintaining the proliferative capability of progenitor cells. Further investigation is needed to explore the potential role of TERT in progenitor cell proliferation. Understanding the mechanism of the regulation of TERT expression could provide valuable insights into cochlear hair cell regeneration and the treatment of hearing impairment.

\section{Acknowledgements}

This study was supported by the National Natural Science Foundation of China (grant no. 81271069) and the National Natural Science Foundation of Major Projects Overseas of China (grant no. 81120108008).

\section{References}

1. White PM, Doetzlhofer A, Lee YS, Groves AK and Segil N: Mammalian cochlear supporting cells can divide and trans-differentiate into hair cells. Nature 441: 984-987, 2006

2. Sinkkonen ST, Chai R, Jan TA, Hartman BH, Laske RD, Gahlen F, Sinkkonen W, Cheng AG, Oshima K and Heller S: Intrinsic regenerative potential of murine cochlear supporting cells. Sci Rep 1: 26, 2011.
3. Shi F, Kempfle JS and Edge AS: Wnt-responsive Lgr5-expressing stem cells are hair cell progenitors in the cochlea. J Neurosci 32: 9639-9648, 2012.

4. Diensthuber M, Oshima K and Heller S: Stem/progenitor cells derived from the cochlear sensory epithelium give rise to spheres with distinct morphologies and features. J Assoc Res Otolaryngol 10: 173-190, 2009.

5. Oshima K, Grimm CM, Corrales CE, Senn P, Martinez Monedero R, Géléoc GS, Edge A, Holt JR and Heller S: Differential distribution of stem cells in the auditory and vestibular organs of the inner ear. J Assoc Res Otolaryngol 8: 18-31, 2007.

6. Collins K and Mitchell JR: Telomerase in the human organism. Oncogene 21: 564-579, 2002.

7. Choi J, Southworth LK, Sarin KY, Venteicher AS, Ma W, Chang W, Cheung P, Jun S, Artandi MK, Shah N, et al: TERT promotes epithelial proliferation through transcriptional control of a Myc- and Wnt-related developmental program. PLoS Genet 4: e10, 2008.

8. Sarin KY, Cheung P, Gilison D, Lee E, Tennen RI, Wang E, Artandi MK, Oro AE and Artandi SE: Conditional telomerase induction causes proliferation of hair follicle stem cells. Nature 436: 1048-1052, 2005.

9. Gage FH: Mammalian neural stem cells. Science 287: 1433-1438, 2000.

10. Chai R, Kuo B, Wang T, Liaw EJ, Xia A, Jan TA, Liu Z, Taketo MM, Oghalai JS, Nusse R, et al: Wnt signaling induces proliferation of sensory precursors in the postnatal mouse cochlea. Proc Natl Acad Sci USA 109: 8167-8172, 2012.

11. Li W, Wu J, Yang J, Sun S, Chai R, Chen ZY and Li H: Notch inhibition induces mitotically generated hair cells in mammalian cochleae via activating the Wnt pathway. Proc Natl Acad Sci USA 112: 166-171, 2014.

12. Harley CB and Villeponteau B: Telomeres and telomerase in aging and cancer. Curr Opin Genet Dev 5: 249-255, 1995.

13. Huffman KE, Levene SD, Tesmer VM, Shay JW and Wright WE: Telomere shortening is proportional to the size of the G-rich telomeric 3'-overhang. J Biol Chem 275: 19719-19722, 2000.

14. Schneider RP, Garrobo I, Foronda M, Palacios JA, Marion RM, Flores I, Ortega S and Blasco MA: TRF1 is a stem cell marker and is essential for the generation of induced pluripotent stem cells. Nat Commun 4: 1946, 2013.

15. Okamoto K, Bartocci C, Ouzounov I, Diedrich JK, Yates JR III and Denchi EL: A two-step mechanism for TRF2-mediated chromosome-end protection. Nature 494: 502-505, 2013.

16. Miller AS, Balakrishnan L, Buncher NA, Opresko PL and Bambara RA: Telomere proteins POT1, TRF1 and TRF2 augment long-patch base excision repair in vitro. Cell Cycle 11: 998-1007, 2012.

17. McKerlie M, Lin S and Zhu XD: ATM regulates proteasome-dependent subnuclear localization of TRF1, which is important for telomere maintenance. Nucleic Acids Res 40: 3975-3989, 2012.

18. Xie Y, Zhao X, Jia H and Ma B: Derivation and characterization of goat fetal fibroblast cells induced with human telomerase reverse transcriptase. In Vitro Cell Dev Biol Anim 49: 8-14, 2013.

19. Wu XQ, Huang C, He X, Tian YY, Zhou DX, He Y, Liu XH and Li J: Feedback regulation of telomerase reverse transcriptase: New insight into the evolving field of telomerase in cancer. Cell Signal 25: 2462-2468, 2013.

20. Singhapol C, Pal D, Czapiewski R, Porika M, Nelson G and Saretzki GC: Mitochondrial telomerase protects cancer cells from nuclear DNA damage and apoptosis. PLoS One 8: e52989, 2013.

21. Plantinga MJ, Pascarelli KM, Merkel AS, Lazar AJ von Mehren M, Lev D and Broccoli D: Telomerase suppresses formation of ALT-associated single-stranded telomeric C-circles. Mol Cancer Res 11: 557-567, 2013.

22. Cong Y and Shay JW: Actions of human telomerase beyond telomeres. Cell Res 18: 725-732, 2008.

23. Ahmed S, Passos JF, Birket MJ, Beckmann T, Brings S, Peters H, Birch-Machin MA, von Zglinicki T and Saretzki G: Telomerase does not counteract telomere shortening but protects mitochondrial function under oxidative stress. J Cell Sci 121: 1046-1053, 2008.

24. Hao LY, Armanios M, Strong MA, Karim B, Feldser DM, Huso D and Greider CW: Short telomeres, even in the presence of telomerase, limit tissue renewal capacity. Cell 123: 1121-1131, 2005.

25. Whitlon DS: E-cadherin in the mature and developing organ of Corti of the mouse. J Neurocytol 22: 1030-1038, 1993.

26. Senn P, Oshima K, Teo D, Grimm C and Heller S: Robust postmortem survival of murine vestibular and cochlear stem cells. J Assoc Res Otolaryngol 8: 194-204, 2007. 